\title{
131.
}

\section{NOUVELLES RECHERCHES SUR LES COVARIANTS.}

[From the Journal für die reine und angewandte Mathematik (Crelle), tom. xLVII. (1854). pp. 109-125.]

JE me sers de la notation

pour représenter la fonction

$$
\left(a_{0}, a_{1}, \ldots a_{n}\right)(x, y)^{n}
$$

$$
a_{0} x^{n}+\frac{n}{1} a_{1} x^{n-1} y+\ldots+a_{n} y^{n}:
$$

en supposant que les coefficients $a_{0}^{\prime}, a_{1}^{\prime} \& c$. soient donnés par l'équation

$$
\left(a_{0}, a_{1}, \ldots a_{n}\right)\left(\lambda x+\mu y, \lambda^{\prime} x+\mu^{\prime} y\right)^{n}=\left(a_{0}^{\prime}, a_{1}^{\prime}, \ldots a_{n}{ }^{\prime}\right)(x, y)^{n},
$$

supposée identique par rapport à $x, y$, soit $\phi\left(a_{0}, a_{1}, \ldots a_{n} ; x, y\right)$ une fonction des coefficients et des variables, telle que

$$
\phi\left(a_{0}{ }^{\prime}, a_{1}{ }^{\prime}, \ldots a_{n}{ }^{\prime} ; \quad x, y\right)=\left(\lambda \mu^{\prime}-\lambda^{\prime} \mu\right)^{p} \phi\left(a_{0}, a_{1}, \ldots a_{n} ; \lambda x+\mu y, \lambda^{\prime} x+\mu^{\prime} y\right) ;
$$

cette fonction $\phi$ sera généralement un Covariant, et dans le cas particulier où $\phi$ est fonction des seuls coefficients, un Invariant de la fonction donnée.

Je suppose d'abord que les nouveaux coefficients soient donnés par l'équation

$$
\left(a_{0}, a_{1}, \ldots a_{n}\right)(x+\lambda y, y)^{n}=\left(a_{0}{ }^{\prime}, a_{1}{ }^{\prime}, \ldots a_{n}{ }^{\prime}\right)(x, y)^{n} ;
$$

cela donne les relations

$$
\begin{aligned}
a_{0}^{\prime}= & a_{0} \\
a_{1}^{\prime}= & a_{1}+\lambda a_{0} \\
a_{2}^{\prime}= & a_{2}+2 \lambda a_{1}+\lambda^{2} a_{0} \\
& \quad \& \mathrm{c} .
\end{aligned}
$$


Il faut donc que le covariant $\phi$ satisfasse à l'équation

$$
\phi\left(a_{0}^{\prime}, a_{1}^{\prime}, \ldots a_{n}^{\prime} ; x, y\right)=\phi\left(a_{0}, a_{1}, \ldots a_{n} ; x+\lambda y, y\right),
$$

laquelle peut aussi être écrite comme suit:

$$
\phi\left(a_{0}^{\prime}, a_{1}^{\prime}, \ldots a_{n}{ }^{\prime} ; x-\lambda y, y\right)=\phi\left(a_{0}, a_{1}, \ldots a_{n} ; x, y\right) .
$$

De même, en faisant

ce qui donne

$$
\left(a_{0}, a_{1}, \ldots a_{n}\right)(x, \mu x+y)^{n}=\left(a_{0}^{\prime}, a_{1}^{\prime}, \ldots a_{n}^{\prime}\right)(x, y)^{n},
$$

$$
\begin{aligned}
& a_{n}^{\prime}=a_{n}, \\
& a_{n-1}=a_{n-1}+\mu a_{n}, \\
& a_{n-2}{ }^{\prime}=a_{n-2}+2 \mu a_{n-1}+a_{n},
\end{aligned}
$$

\&c.

le covariant $\phi$ doit satisfaire aussi à l'équation

$$
\phi\left(a_{0}^{\prime}, a_{1}^{\prime}, \ldots a_{n}^{\prime} ; \quad x,-\mu x+y\right)=\phi\left(a_{0}, a_{1}, \ldots a_{n} ; x, y\right) ;
$$

et réciproquement, toute fonction $\phi$ homogène par rapport aux coefficients et aussi par rapport aux variables, qui satisfait à ces équations $(X, Y)$, sera un covariant de la fonction donnée.

Examinons d'abord l'équation $(X)$ que je représente par $\phi^{\prime}=\phi$. Soit pour le moment, $a_{1}^{\prime}-a_{1}=\lambda \alpha_{1}, \quad a_{2}^{\prime}-a_{2}=\lambda \alpha_{2}$, \&c., alors on aura, comme à l'ordinaire, l'équation symbolique

$$
\phi^{\prime}=e^{\lambda\left(a_{1} \partial_{1_{1}}+a_{2} \partial a_{2} \ldots+a_{n} \partial_{a_{n}}-y \partial_{z}\right)} \phi,
$$

où les quantités $a_{1}, a_{2}, \&$ c., en tant qu'elles entrent dans $\alpha_{1}, \alpha_{2}$, \&c., ne doivent pas être affectées par les symboles $\partial_{a_{1}}, \partial_{a_{2}}$, \&c. de la différentiation. En substituant les valeurs de $\alpha_{1}, \alpha_{2}, \ldots$, et en ordonnant selon les puissances de $\lambda$, cette équation donne

$$
\phi^{\prime}=e^{\lambda \varphi^{+}+\lambda^{2} \square_{1} \cdots+\lambda n \square_{n-1}-\lambda y \partial_{x}} \phi,
$$

où les symboles $\square, \square_{1}$, \&c. sont donnés par

$$
\begin{aligned}
& \square=a_{0} \partial_{a_{1}}+2 a_{1} \partial_{a_{2}} \ldots+n a_{n-1} \partial_{a_{n}}, \\
& \square_{1}=a_{0} \partial_{a_{2}}+3 a_{1} \partial_{a_{3}} \ldots+\frac{n \cdot n-1}{1.2} a_{n-2} \partial_{a_{n}}, \\
& \vdots \\
& \square_{n-1}=a_{0} \partial_{a_{n}}
\end{aligned}
$$

et les quantités $a_{1}, a_{2}$, \&c., en tant qu'elles entrent dans les symboles $\square, \square_{1}$, \&c., ne doivent pas être affectées par les symboles $\partial_{a_{1}}, \partial_{a_{2}}$, \&c. de la différentiation. Il est assez remarquable que l'équation symbolique peut aussi être écrite sous la forme plus simple

$$
\phi^{\prime}=e^{\lambda\left(\square-y \partial_{x}\right)} \phi,
$$


où les quantités $a_{1}, a_{2}, \ldots$, en tant qu'elles entrent dans le symbole $\square$, sont censées affectées des symboles $\partial_{a_{1}}, \partial_{a_{2}}$, \&c. de la différentiation; de manière que dans le développement, $\square^{2} . \phi$ par exemple, signifie $\square . \square \phi$, et ainsi de suite. Je ne m'arrête pas sur ce point, parce que pour ce que je vais démontrer de plus important, il suffit de faire attention à la première puissance de $\lambda$. D'ailleurs l'intelligibilité des équations dont il s'agit, sera facilitée en faisant les développements et en comparant les puissances correspondantes de $\lambda$. Cela donne par exemple:

$$
\square^{2}=\square^{2}+2 \square_{1}, \quad \square^{3}=\square^{3}+3 \square \square_{1}+6 \square_{2}, \& c .
$$

où les symboles $\square^{2}, \square^{3}$ \&c. à gauche de ces équations dénotent la double, triple, \&c. répétition de l'opération $\square$, tandis qu'à côté droit des équations, les quantités $a_{1}, a_{2}, \ldots$ \&c., en tant qu'elles entrent dans les symboles $\square, \square_{1}$, \&c. sont censées ne pas être affectées des symboles $\partial_{a_{1}}, \partial_{a_{2}}$, \&c. de la différentiation. Dans la suite, si le contraire n'est pas dit, je me servirai des expressions $\square^{2}, \square^{3}$, \&c. pour dénoter les répétitions de l'opération, et de même pour les combinaisons de deux ou de plusieurs symboles.

Cela étant, l'équation $\phi^{\prime}=e^{\lambda\left(\square-y \theta_{x}\right)} \phi=\phi$ donne

$$
\phi=\left\{1+\lambda\left(\square-y \partial_{x}\right)+\frac{\lambda^{2}}{1.2}\left(\square-y \partial_{x}\right)^{2}+\ldots\right\} \phi,
$$

où $\left(\square-y \partial_{x}\right)^{2} \cdot \phi$ (je le répète) équivaut à $\left(\square-y \partial_{x}\right) \cdot\left(\square-y \dot{\partial}_{x}\right) \phi$; et ainsi de suite. Il faut d'abord que le coefficient de $\lambda$ s'évanouisse, ce qui donne $\left(\square-y \partial_{x}\right) \phi=0$; et cette condition étant satisfaite, les coefficients des puissances supérieures s'évanouissent d'ellesmêmes; c'est-à-dire, l'équation $(X)$ sera satisfaite en supposant que $\phi$ satisfait à l'équation aux différences partielles $\left(\square-y \partial_{x}\right) \phi=0$.

En posant

$$
\begin{aligned}
& \dot{\square}=a_{n} \partial_{a_{n-1}}+2 a_{n-1} \partial_{a_{n-2}} \ldots+n a_{1} \partial_{a_{0}}, \\
& \dot{\square}_{1}=a_{n} \partial_{a_{n-2}}+3 a_{n-1} \partial_{a_{n-3}} \ldots+\frac{n(n-1)}{1.2} a_{2} \partial_{a_{0}}, \\
& \vdots \\
& \dot{\square}_{n-1}=a_{n} \partial_{a_{0}},
\end{aligned}
$$

on fera un raisonnement analogue par rapport à l'équation $(Y)$; et il sera ainsi démontré que $\phi$ doit satisfaire aussi à l'équation à différences partielles $\left(\dot{\square}-x \partial_{\gamma}\right) \phi=0$; donc enfin, on a le suivant

ThÉoRème. Tout covariant $\phi$ de la fonction

$$
\left(a_{0}, a_{1}, \ldots a_{n}\right)(x, y)^{n},
$$

satisfait aux deux équations à différences partielles

où

$$
\left(\square-y \partial_{x}\right) \phi=0, \quad\left(\dot{\square}-x \partial_{y}\right) \phi=0,
$$

$$
\begin{aligned}
& \square=a_{0} \partial_{a_{1}}+2 a_{1} \partial_{\dot{a}_{2}} \ldots+n a_{n-1} \partial_{a_{n}}, \\
& \dot{\square}=n a_{1} \partial_{a_{0}}+(n-1) a_{2} \partial_{a_{1}} \ldots+a_{n} \partial_{a_{n-1}} ;
\end{aligned}
$$


et réciproquement toute fonction, homogène par rapport aux coefficients et par rapport aux variables, qui satisfait à ces équations, est un covariant de la fonction donnée.

Par exemple, l'invariant $\phi=a c-b^{2}$ de la fonction $a x^{2}+2 b x y+c y^{2}$ satisfait aux équations

$$
\left(a \partial_{b}+2 b \partial_{c}\right) \phi=0, \quad\left(2 b \partial_{a}+c \partial_{b}\right) \phi=0,
$$

et le covariant $\phi=\left(a c-b^{2}\right) x^{2}+(a \partial-b c) x y+\left(b \partial-c^{2}\right) y^{2}$ de la fonction $a x^{3}+3 b x^{2} y+3 c x y^{2}+d y^{3}$ satisfait aux équations

$$
\left(a \partial_{b}+2 b \partial_{c}+3 c \partial_{d}-y \partial_{x}\right) \phi=0, \quad\left(3 d \partial_{c}+2 c \partial_{b}+b \partial_{a}-x \partial_{y}\right) \phi=0 .
$$

Il est clair qu'en ne considérant que les fonctions qui restent les mêmes en prenant dans un ordre inverse les coefficients $a_{0}, a_{1}, \ldots a_{n}$ et les variables $x, y$, respectivement, les covariants seront définis par l'une ou l'autre des équations $(A)$, et qu'il n'est plus nécessaire de considérer les deux équations. Cela posé, on trouve assez facilement les conariants par la méthode des coefficients indéterminés. Mais il y a à remarquer une circonstance de la plus grande importance dans cette théorie, savoir, que l'on obtient de cette manière un nombre d'équations plus grand qu'il n'en faut pour déterminer les coefficients dont il s'agit. Ces équations cependant, étant liées entre elles, se réduisent au nombre nécessaire d'équations indépendantes.

Cherchons par exemple pour la fonction $a x^{3}+3 b x^{2} y+3 c x y^{2}+d y^{3}$ un invariant $\phi$ de la forme

$$
\phi=A a^{2} d^{2}+B a b c d+C a c^{3}+C b^{3} d+D b^{2} c^{2},
$$

contenant les quatre coefficients indéterminés $A, B, C, D$. En substituant dans l'équation $\left(a \partial_{b}+2 b \partial_{c}+3 c \partial_{d}\right) \phi=0$, on obtient

$$
(3 C+2 B) a b^{2} d+(3 B+6 C+2 D) a b c^{2}+(6 A+B) a c^{2} d+(3 C+4 D) b^{3} c=0 ;
$$

or les quatre équations données par cette condition, se réduisent à trois équations indépendantes, de sorte qu'en faisant par exemple $A=-1$, les autres coefficients seront déterminés, et l'on obtient le résultat connu :

$$
\phi=-a^{2} d^{2}+6 a b c d-4 a c^{3}-4 b^{3} d+3 b^{2} c^{2} .
$$

La circonstance mentionnée ci-dessus s'oppose à résoudre de la manière dont il s'agit, le problème de trouver le nombre des invariants d'un ordre donné: problème qui a toujours bravé mes efforts.

Avant d'entamer la solution des équations $(A)$, je vais démontrer quelques propriétés générales des covariants, et des invariants. Pour abréger, je me servirai du mot pesanteur, en disant que les coefficients $a_{0}, a_{1}$, \&c., ont respectivement les pesanteurs $0-\frac{1}{2} n, 1-\frac{1}{2} n$, \&c., que les variables $x, y$ ont respectivement les pesanteurs $\frac{1}{2},-\frac{1}{2}$, et que la pesanteur 
d'un produit est égale à la somme des pesanteurs des facteurs. Cela posé, je dis que tout covariant est composé de termes dont chacun à la pesanteur zéro. Pour démontrer cela, j'écris :

$$
\begin{aligned}
& \left(\square+-y \partial_{x}\right)\left(\dot{\square}-x \partial_{y}\right)=\square \dot{\square}-y \partial_{x} \dot{\square}-x \partial_{y} \square+x y \partial_{x} \partial_{y}+y \partial_{y} \text {, } \\
& \left(\dot{\square}-x \partial_{y}\right)\left(\square-y \partial_{x}\right)=\dot{\square} \square-y \partial_{x} \dot{\square}-x \partial_{y} \square+x y \partial_{x} \partial_{y}+x \partial_{x} ;
\end{aligned}
$$

cela donne

$$
\left(\square+-y \partial_{x}\right)\left(\dot{\square}-x \partial_{y}\right)-\left(\dot{\square}-x \partial_{y}\right)\left(\underline{\varphi}-y \partial_{x}\right)=\underline{\square} \dot{\square}-\dot{\square} \square+y \partial_{y}-x \partial_{x} ;
$$

or, en faisant attention aux valeurs de $\square, \dot{\square}$, savoir

$$
\begin{aligned}
& \dot{\square} \dot{\square}=(\dot{\square} \dot{\square})+n a_{0} \partial_{a_{0}}+2(n-1) a_{1} \partial_{a_{1}} \ldots+n 1 a_{n-1} \partial_{a_{n-1}}, \\
& \dot{\square} \square=(\dot{\square} \square)+\quad
\end{aligned}
$$

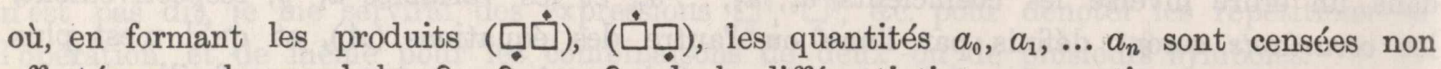
affectées par les symboles $\partial_{a_{0}}, \partial_{a_{1}}, \ldots \partial_{a_{n}}$ de la différentiation, on en tire

$$
\begin{aligned}
\square \dot{ப}-\dot{\square} \square & =n a_{0} \partial_{a_{0}}+(n-2) a_{1} \partial_{a_{1}} \ldots-n a_{n} \partial_{a_{n}} \\
& =-2\left\{\left(0-\frac{1}{2} n\right) a_{0} \partial_{a_{0}}+\left(1-\frac{1}{2} n\right) a_{1} \partial_{a_{1}} \ldots+\left(n-\frac{1}{2} n\right) a_{n} \partial_{a_{n}}\right\}=-2 \Theta,
\end{aligned}
$$

en représentant par $\Theta$ l'expression symbolique entre les crochets. De là enfin on obtient:

$$
\left(\square-y \partial_{x}\right)\left(\dot{\square}-x \partial_{y}\right)-\left(\dot{\square}-x \partial_{y}\right)\left(\square-y \partial_{x}\right)=-2\left(\Theta+\frac{1}{2} x \partial_{x}-\frac{1}{2} y \partial_{y}\right) \text {. }
$$

Or en supposant les deux parties de cette équation symbolique appliquées au covariant $\phi_{1}$, la partie gauche de l'équation s'évanouit en vertu des équations $(A)$ et l'équation se réduit à

$$
\left(\Theta+\frac{1}{2} x \partial_{x}-\frac{1}{2} y \partial_{y}\right) \phi=0 ;
$$

ce qui est une nouvelle équation à différences partielles, à laquelle satisfait le covariant $\phi$. Il est aisé de voir que cette équation exprime le théorème énoncé ci-dessus, savoir que tout covariant est composé de termes de la pesanteur zéro.

Il suit de là, en considérant un covariant

$$
\phi=\left(A_{0}, A_{1}, \ldots A_{s}\right)(x, y)^{s}
$$

qu'un coefficient quelconque $A_{i}$ aura la pesanteur $i-\frac{1}{2} s$, ou bien que les pesanteurs forment une progression arithmétique aux différences 1 , et dont les termes extrèmes sont $-\frac{1}{2} s,+\frac{1}{2} s$.

Substituons maintenant cette valeur de $\phi$ dans les équations $(A)$. La première équation donne d'abord:

$$
\square A_{0}=0, \quad \square A_{1}=A_{0}, \quad \square A_{2}=2 A_{1}, \ldots \square A_{s}=s A_{s-1} .
$$

Cela est un système qui équivaut aux deux équations

$$
\square^{s} \cdot A_{s}=0, \quad \phi=y^{s} \cdot e^{\square \frac{x}{y}} \cdot A_{s} .
$$


De même, la seconde équation donne

$$
\dot{\square} A_{s}=0, \quad \dot{\square} A_{8-1}=A_{8}, \quad \dot{\square} A_{s-2}=2 A_{s-1}, \ldots \quad \dot{\square} A_{0}=s A_{1}:
$$

système qui équivaut aux deux équations

$$
\dot{\square}^{s+1} A_{0}=0, \quad \phi=x^{s} e^{\dot{\square} \frac{y}{x}} \cdot A_{0} .
$$

On voit que $A_{0}$ satisfait aux deux équations

$$
\square A_{0}=0, \quad \dot{\square}^{s+1} A_{0}=0,
$$

et en supposant que cette quantité soit connue, on trouve les autres coefficients $A_{1}, A_{2}, \ldots, A_{8}$ par la seule différentiation, au moyen des équations $(\beta)$. Or cela étant, je dis que les équations $(\alpha)$ seront satisfaites d'elles-mêmes. En effet: des équations

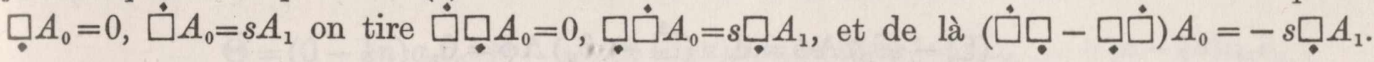

Or nous avons déjà vu que $\dot{\sqcup} \square-\square \dot{\square}=2 \Theta$, et l'équation $(B)$ donne $\Theta \cdot A_{0}+\frac{1}{2} s \cdot A_{0}=0$ : donc l'équation ( $\dot{\square} \square-\square \underline{\square}) A_{0}=-s \square \dot{A}_{1}$ se réduit à $A_{0}=\square A_{1}$ : équation du système (a). De la même manière on obtient les autres équations de ce système. On peut dire que l'on aurait pu déterminer également le coefficient $A_{s}$ au moyen des équations

$$
\dot{\square} A_{s}=0, \quad \square^{s} \cdot A_{s}=0,
$$

et de là les coefficients $A_{s-1}, \ldots A_{0}$ par les équations $(\alpha)$.

Prenons par exemple un covariant $\left(A_{0}, A_{1}, A_{2}\right)(x, y)^{2}$ de la fonction cubique $a x^{3}+3 b x^{2} y+3 c x y^{2}+d y^{3} . \quad A_{0}$ doit satisfaire aux deux équations

$$
\left(a \partial_{b}+2 b \partial_{c}+3 c \partial_{d}\right) A_{0}=0, \quad\left(3 b \partial_{a}+2 c \partial_{b}+d \partial_{c}\right)^{3} A_{0}=0 .
$$

Ces équations sont en effet satisfaites en mettant $A_{0}=a c-b^{2}$. On a donc les équations

$$
2 A_{1}=\left(3 b \partial_{a}+2 c \partial_{b}+d \partial_{c}\right) A_{0}, \quad A_{2}=\left(3 b \partial_{a}+2 c \partial_{b}+d \partial_{c}\right) A_{1},
$$

pour déterminer $A_{1}, A_{2}$; ce qui donne $2 A_{1}=a d-b c, A_{2}=b d-c^{2}$, et on est conduit ainsi au covariant mentionné ci-dessus, savoir à

$$
\left(a c-b^{2}\right) x^{2}+(a d-b c) x y+\left(b d-c^{2}\right) y^{2} .
$$

Soit maintenant

$$
x^{n} \partial_{a_{n}}-x^{n-1} y \partial_{a_{n-1}} \cdots \pm y^{n} \partial_{a_{0}}=\Lambda,
$$

on aura

$$
\square \Lambda=(\square \Lambda), \quad \Lambda \square=(\Lambda \square)-y \frac{\partial \Lambda}{\partial x},
$$

C. II. 
où dans $(\square \Lambda),(\Lambda \square)$ les quantités $a_{0}, a_{1}, \ldots$ sont censées non affectées par les symboles $\partial_{a_{1}}, \partial_{a_{2}}, \& c$. de la différentiation. Cela donne

$$
\square \Lambda-\Lambda \square=y \frac{d \Lambda}{\partial x} \text {. }
$$

Or $\partial_{x} \Lambda-\Lambda \partial_{x}=\frac{d \Lambda}{\partial x}$, donc :

$$
\left(\square-y \partial_{x}\right) \Lambda=\Lambda\left(\square-y \partial_{x}\right),
$$

et de même:

$$
\left(\dot{\square}-x \partial_{y}\right) \Lambda=\Lambda\left(\dot{\square}-x \partial_{y}\right)
$$

Appliquons ces deux équations symboliques à un covariant $\phi$. Les termes à droite s'évanouissent à cause des équations $(A)$, et l'on obtient les deux équations

$$
\left(\square+-y \partial_{x}\right) \Lambda \phi=0, \quad\left(\dot{\square}-x \partial_{y}\right) \Lambda \phi=0,
$$

c'est-à-dire: $\Lambda \phi$ sera aussi un covariant de la fonction donnée. Par exemple de linvariant

$$
\phi=-a^{2} d^{2}+6 a b c d-4 a c^{3}-4 b^{3} d+3 b^{2} c^{2},
$$

on tire le covariant

$$
\left(x^{3} \partial_{d}-x^{2} y^{2} \partial_{c}+x y^{2} \partial_{b}-y^{3} \partial_{a}\right) \phi ;
$$

savoir :

$$
\begin{aligned}
& \left(-a^{2} d+3 a b c-2 b^{3}\right) x^{3} \\
- & 3\left(a b d-2 a c^{2}+b^{2} c\right) x^{2} y \\
+ & 3\left(a c d-2 b^{2} d+b c^{2}\right) x y^{2} \\
- & \left(-a d^{2}+3 a c d-2 c^{3}\right) y^{3}
\end{aligned}
$$

résultat déjà connu.

Essayons maintenant à intégrer les équations $(A)$; savoir:

$$
\left(\square-y \partial_{x}\right) \phi=0, \quad\left(\dot{\square}-x \partial_{y}\right) \phi=0 .
$$

Pour intégrer la première, je reviens à une notation dont je me suis déjà servi dans ce mémoire et j'écris

$$
\begin{aligned}
& a_{0}^{\prime}=a_{0}, \\
& a_{1}^{\prime}=a_{1}+\lambda a_{0}, \\
& a_{2}^{\prime}=a_{2}+2 \lambda a_{1}+\lambda^{2} a_{0}, \\
& \vdots \\
& a_{n}^{\prime}=a_{n}+n \lambda a_{n-1} \ldots+\lambda^{n} a_{0} .
\end{aligned}
$$


En faisant $\lambda=-\frac{a_{1}}{a_{0}}$, ce qui donne $a_{0}^{\prime}=0$, on voit sans peine que l'on satisfera à l'équation, en mettant pour $\phi$ une fonction quelconque de quantités $a_{0}^{\prime}, a_{1}^{\prime}, \ldots a_{n}$, $x+\lambda y, y$; le nombre de ces quantités étant $n+2$. Et cela est la solution générale de l'équation.

Ce résultat doit être substituée dans la seconde équation, savoir dans $\left(\dot{\square}-x \partial_{y}\right) \phi=0$. Pour cela, imaginons que les quantités $a_{0}, a_{1}, \ldots a_{n}, x, y$ soient exprimées en fonction de $a_{0}^{\prime}, a_{2}^{\prime}, \ldots a_{n}^{\prime}, x, y$ et $a_{1}$; puisque $\phi$ est fonction des seules quantités $a_{0}^{\prime}, a_{2}^{\prime} \ldots a_{n}{ }^{\prime}$, $x, y$, l'équation résultante doit être satisfaite, quelle que soit la valeur de $a_{1}$. Or on trouve que cette équation résultante a la forme $L+M a_{1}=0$ : done il faut qu'on ait à la fois les deux équations $L=0, M=0$. (Je renvoie à une note les détails de la réduction.) En dernière analyse, et en remettant dans les équations $L=0, M=0$ les quantités $a_{0}, a_{2}, \ldots, a_{n}$ au lieu de $a_{0}^{\prime}, a_{2}^{\prime}, \ldots, a_{n}^{\prime}$, je trouve les résultats suivants très simples, savoir, en écrivant

$$
\begin{aligned}
& \bar{\Theta}=\left(0-\frac{1}{2} n\right) a_{0} \partial_{a_{0}}+\quad\left(2-\frac{1}{2} n\right) a_{2} \partial_{a_{2}}+\left(3-\frac{1}{2} n\right) a_{3} \partial_{a_{3}} \ldots+\left(n-\frac{1}{2} n\right) a_{n} \partial_{a_{n}}: \\
& \cdot \square=\quad 3 a_{2} \partial_{a_{3}}+4 a_{3} \partial_{a_{4}} \cdots+n a_{n-1} \partial_{a_{n}}, \\
& \text { · } \dot{\square}=(n-2) a_{3} \partial_{a_{2}}+(n-3) a_{4} \partial_{a_{3}} \ldots \quad+a_{n} \partial_{a_{n-1}} \text {. }
\end{aligned}
$$

Les équations dont il s'agit sont

$$
\begin{array}{r}
\left\{(n-1) a_{2}\left(\cdot \square-y \partial_{x}\right)-a_{0}\left(\cdot \square-x \partial_{y}\right)\right\} \bar{\phi}=0, \\
\left(\bar{\Theta}+\frac{1}{2} x \partial_{x}-\frac{1}{2} y \partial_{y}\right) \bar{\phi}=0,
\end{array}
$$

et il y a à remarquer qu'on obtient l'équation $(C)$ en éliminant entre les équations (A) le terme $\partial_{a_{1}} \phi$; et puis, en mettant $a_{1}=0$, on tire l'équation $(D)$ de l'équation $(B)$, en y mettant de même $a_{1}=0$. Il y a à remarquer aussi que la fonction $\bar{\phi}$ qui satisfait aux équations $(C, D)$, est ce que devient un covariant quelconque $\phi$, en y mettant $a_{1}=0$. On obtient d'abord la valeur générale en changeant $a_{0} ; a_{2}, \ldots, a_{n}$ en $a_{0}^{\prime}, a_{2}^{\prime}, \ldots, a_{n}^{\prime}$, et en mettant après pour ces quantités leurs valeurs en termes de $a_{0}, a_{1}, a_{2}, \ldots, a_{n}$. La solution du problème des covariants serait donc effectuée si l'on pourrait intégrer les équations $(C, D)$.

Or la quantité $a_{0}$ entre dans l'équation $(C)$ comme constante, et l'on voit sans peine que cette équation pourra être intégrée en mettant $a_{0}=1$; puis, en écrivant dans le résultat $\frac{a_{2}}{a_{0}}, \frac{a_{3}}{a_{0}}, \ldots \frac{a_{n}}{a_{0}}$ au lieu de $a_{2}, a_{3}, \ldots a_{n}$, et en multipliant par une puissance quelconque de $a_{0}$, le résultat ainsi obtenu, serait composé de termes de la même pesanteur; et en choisissant convenablement la puissance de $a_{0}$, on pourrait faire en sorte que ces termes fussent de la pesanteur zéro. Mais l'équation $(D)$ ne fait qu'exprimer que la fonction $\bar{\phi}$ est composée de termes de la pesanteur zéro; le résultat obtenu de la manière dont il s'agit, satisfera donc par lui-même à l'équation $(D)$, et il est permis de ne faire attention qu'à l'équation $(C)$. Dans la pratique on intégrera cette 
équation en ayant soin de faire en sorte que les solutions soient de la pesanteur zéro, ce qui peut être effectué en multipliant par une puissance convenablement choisie de $a_{0}$. Et puisqu'en faisant abstraction de cette quantité $a_{0}$, l'équation $(C)$ contient $n+1$ quantités variables, savoir $a_{2}, a_{3}, \ldots, a_{n}, x, y$, la fonction $\bar{\phi}$ sera une fonction arbitraire de $n$ quantités; et en supposant que cette fonction ne. contienne pas les variables $x, y$ (cas auquel $\bar{\phi}$ serait ce que deviendrait un invariant quelconque en y mettant $a_{1}=0$ ), $\bar{\phi}$ sera une fonction arbitraire de $n-2$ quantités.

La même chose sera évidemment vrai, si l'on rétablit la valeur générale de $a_{1}$ : donc tout invariant sera une fonction d'un nombre $n-2$ d'invariants, que l'on pourra prendre pour primitifs; et tout covariant sera une fonction de ces invariants primitifs de la fonction donnée (laquelle est évidemment un de ses propres covariants), et d'un autre covariant que l'on peut prendre pour primitif. Cela ne prouve nullement (ce qui est néanmoins vrai pour les invariants, à ce que je crois) que tout invariant est une fonction rationnelle et intégrale de $n-2$ invariants convenablement choisis, et que tout covariant est une fonction rationnelle et intégrale (ce qui en effet n'est pas vrai) de ces invariants, de la fonction donnée, et d'un covariant convenablement choisi.

Le cas $n=2$ fait dans cette théorie une exception. On sait qu'il existe dans ce cas un invariant, savoir $a c-b^{2}$ qui, selon la théorie générale, ne doit pas exister, et il n'existe pas de covariant, hormis la fonction donnée elle-même. Or cette particularité peut être aisément expliquée.

Le cas $n=3$ rentre, comme cela doit être, dans la théorie générale. En effet, il existe dans ce cas un invariant, savoir la fonction $-a^{2} d^{2}+6 a b c d+4 a c^{3}-4 b^{3} d+3 b^{2} c^{2}$ ci-dessus trouvée, et tout covariant de la fonction peut être exprimé par cet invariant de la fonction donnée elle-même, et par le covariant $\left(a c-b^{2}\right) x^{2}+(a d-b c) x y+\left(b d-c^{2}\right) y^{2}$ ci-dessus trouvé. Il en est ainsi par exemple pour le covariant de troisième ordre par rapport aux variables et aux coefficients; car en représentant par $\Phi$ le covariant dont il s'agit, par $H$ le covariant du second ordre, par $u$ la fonction donnée $a x^{3}+3 b x^{2} y+3 c x y^{2}+d y^{3}$ et par $\nabla$ l'invariañt, on obtient l'équation identique $\Phi^{2}+\square u^{2}=-4 H^{3}$. Je fais mention de cette équation, parce que je crois qu'elle n'est pas généralement connue.

Je vais donner maintenant quelques exemples des équations ( $C$ et $D$ ). Soit d'abord $n=3$, et supposons que $\bar{\phi}$ ne contienne pas les variables $x, y: \bar{\phi}$ sera une fonction de $a, c, d$, et les équations reviendront à

$$
\left(6 c^{2} \partial_{d}-a d \partial_{c}\right) \bar{\phi}=0, \quad\left(-3 a \partial_{a}+c \partial_{c}+3 d \partial_{d}\right) \bar{\phi}=0 .
$$

Les quantités $a c^{3}, a^{2} d^{2}$, dont chacune est de la pesanteur zéro, satisfont par là à la seconde équation, et en mettant $\bar{\phi}=A a^{2} d^{2}+C a c^{3}$, on obtient $4 A-C=0$, en vertu de la première équation; ou en faisant $A=-1$, cela donne $C=-4$; de là on tire $\bar{\phi}=-a^{2} d^{2}-4 a c^{3}$, et la solution générale est $\bar{\phi}=F\left(-a^{2} d^{2}-4 a c^{3}\right), F$ étant une fonction quelconque. La formule plus générale $\bar{\phi}=F\left(a,-a^{2} d^{2}-4 a c^{3}\right)$ satisferait sans doute à la 
première équation, mais pour que cette valeur satisfasse à la seconde équation, il faut que la quantité $a$, en tant qu'elle n'est pas contenue dans $-a^{2} d^{2}-4 a c^{3}$, disparaisse. Ainsi la valeur donnée ci-dessus, savoir $\bar{\phi}=F\left(-a^{2} d^{2}-4 a c^{3}\right)$, est la solution la plus générale des deux équations.

Écrivons $a, c-\frac{b^{2}}{a}, d-\frac{3 b c}{a}+\frac{2 b^{3}}{a^{2}}$ au lieu de $a, c, d$, et $\phi$ au lieu de $\bar{\phi}$, nous obtenons:

$$
\phi=F\left(-a^{2} d^{2}+6 a b c d-4 a c^{3}-4 b^{3} d+3 b^{2} c^{2}\right) ;
$$

ce qui est l'expression la plus générale des invariants de la fonction $a x^{3}+3 b x^{2} y+3 c x y^{2}+y^{3}$, et l'on voit que tous ces invariants sont fonctions d'une seule quantité que nous avons prise ci-dessus pour l'invariant de la fonction de troisième ordre dont il s'agit.

Soit encore $n=4, \bar{\phi}$ sera une fonction de $a, c, d$, e qui satisfait aux équations

$$
\begin{array}{r}
\left\{2 a d \partial_{c}+\left(a e-9 c^{2}\right) \partial_{d}-12 c d \partial_{e}\right\} \bar{\phi}=0, \\
\left\{-2 a \partial_{a}+d \partial_{d}+2 e \partial_{e}\right\} \bar{\phi}=0,
\end{array}
$$

dont la solution générale est $\bar{\phi}=F\left(a e+3 c^{2}\right.$, $\left.a c e-a d^{2}-c^{3}\right), F$ étant une fonction quelconque. On voit par là qu'il n'existe que les invariants indépendants $a e-4 c d+3 c^{2}$, $a c e+2 b c d-a d^{2}-b^{2} e-c^{3}$. Ce résultat est connu depuis longtemps.

Soit enfin $n=5, \bar{\phi}$ sera une fonction de $a, c, d, e, f$ qui satisfait aux équations

$$
\begin{array}{r}
\left\{3 a d \partial_{c}+\left(2 a e-12 c^{2}\right) \partial_{d}+(a f-16 c d) \partial_{e}-20 c e \partial_{f}\right\} \bar{\phi}=0 \\
\left\{-\frac{5}{2} a \partial_{a}-\frac{1}{2} c \partial_{c}+\frac{1}{2} d \partial_{d}+\frac{3}{2} e \partial_{e}+\frac{5}{2} f \partial_{f}\right\} \bar{\phi}=0 .
\end{array}
$$

On sait qu'il y en a une solution de quatrième ordre par rapport aux quantités $a, c, d, e, f$; et en prenant la fonction la plus générale dont les termes ont la pesanteur zéro, on aura:

$$
\bar{\phi}=A a^{2} f^{2}+B a c d f+C a c e^{2}+D a d^{2} e+E c^{3} e+F c^{2} d^{2}:
$$

fonction qui satisfait d'elle-même à la seconde équation. En substituant cette valeur dans la première équation, on trouvera que les coefficients $A, B$, \&c. doivent satisfaire à ces sept équations :

$$
\begin{aligned}
& 2 B+2 C-40 A=0, \quad 3 B+D=0, \quad 3 C+4 D=0, \quad-12 B+E=0, \\
& 9 E-24 D+4 F-32 C-20 B=0, \quad 6 F-16 D=0, \quad-24 F-16 E=0,
\end{aligned}
$$

qui se réduisent cependant (ce que l'on n'aurait pas facilement deviné par la forme des équations) à cinq équations indépendantes. En faisant donc $A=1$, on trouve aisément les autres coefficients $B, C$, \&c. et on obtient ainsi :

$$
\bar{\phi}=a^{2} f^{2}+4 a c d f+16 a c e^{2}-12 a d^{2} e+48 c^{3} e-32 c^{2} d^{2}:
$$

valeur qui peut être tirée d'une formule présentée dans mon mémoire sur les hyperdéterminants, [16], en y faisant $b=0$. 
J'ai donné cet exemple pour faire voir qu'il serait impossible de déduire du nombre supposé connu des coefficients indéterminés qui correspondent à un ordre donné, le nombre des invariants de ce même ordre. Il est donc inutile de pousser plus loin cette discussion.

Note 1 sur l'intégration des équations $(A)$.

En écrivant comme ci-dessus :

$$
\begin{aligned}
& \square=a_{0} \partial_{a_{1}}+2 a_{1} \partial a_{a_{2}} \ldots \quad+n a_{n-1} \partial_{a_{n}}, \\
& \dot{\square}=n a_{1} \partial_{a_{0}}+(n-1) a_{2} \partial_{a_{1}} \ldots+a_{n} \partial_{a_{n-1}},
\end{aligned}
$$

il s'agit de trouver une quantité $\phi$, fonction de $a_{0}, a_{1}, \ldots a_{n}, x$ et $y$ qui satisfasse à la fois aux équations

$$
\begin{aligned}
& \left(\square-y \partial_{x}\right) \phi=0, \\
& \left(\dot{\square}-x \partial_{y}\right) \phi=0 .
\end{aligned}
$$

Pour intégrer ces équations, j'écris, comme plus haut:

$$
\begin{aligned}
& a_{0}^{\prime}=a_{0}, \\
& a_{1}^{\prime}=a_{1}+\lambda a_{0}, \\
& a_{2}^{\prime}=a_{2}+2 \lambda a_{1}+\lambda^{2} a_{0}, \\
& \vdots \\
& a_{n}^{\prime}=a_{n}+n \lambda a_{n-1} \cdots \quad+\lambda^{n} a_{0},
\end{aligned}
$$

et aussi $x^{\prime}=x-\lambda y, y^{\prime}=y$. Cela posé, je fais remarquer d'abord que $\frac{d a_{1}^{\prime}}{d \lambda}=a_{0}{ }^{\prime}, \frac{d a_{2}^{\prime}}{d \lambda}=2 a_{1}^{\prime}$, et ainsi de suite. En considérant $\lambda$ comme fonction quelconque de $a_{0}, a_{1}, \ldots a_{n}$, et en supposant que $\phi$ soit une fonction de $a_{0}^{\prime}, a_{1}^{\prime}, \ldots a_{n}^{\prime}, x^{\prime}, y^{\prime}$, on parvient assez facilement à l'équation identique $\left(\square-y \partial_{x}\right) \phi=(1+\square \lambda)\left(\square^{\prime}-y^{\prime} \partial_{x^{\prime}}\right) \phi$, où $\square^{\prime}$ est ce que devient $\square$, en y écrivant $a_{0}^{\prime}, a_{1}^{\prime}, \ldots a_{n}^{\prime}$ au lieu de $a_{0}, a_{1}, \ldots a_{n}$.

Nous pouvons donc satisfaire à la première équation, en déterminant $\lambda$ au moyen de $1+\square \lambda=0$ : équation qui serait satisfaite en écrivant $\lambda=-\frac{a_{1}}{a_{0}}$, ou, si l'on veut, en déterminant $\lambda$ par $a_{1}^{\prime}=0$. Donc, en supposant toujours que $\lambda$ ait cette valeur, $\phi$ sera une fonction quelconque de $a_{0}^{\prime}, a_{2}^{\prime}, \ldots a_{n}^{\prime}, x^{\prime}, y^{\prime}$, c'est-à-dire d'un nombre $n+2$ de quantités. Ce sera donc là (comme on aurait pu facilement prévoir), la solution générale de la première équation. Or en considérant $\phi$ comme fonction de $a_{0}^{\prime}, a_{2}^{\prime}, \ldots a_{n}{ }^{\prime}$, $x^{\prime}, y^{\prime}$, ou, si l'on veut, de $a_{0}^{\prime}, a_{1}^{\prime}, a_{2}^{\prime}, \ldots a_{n}^{\prime}, x^{\prime}, y^{\prime}$ (où $a_{1}^{\prime}=a_{1}+\lambda a_{0}=0$ ), et en 
substituant cette valeur dans l'équation $\left(\dot{\square}-x \partial_{y}\right) \phi=0$, on voit d'abord que la variation de la quantité $\lambda$ fournit au résultat le terme

$$
\left(n a_{1} \frac{d \lambda}{d a_{0}}+(n-1) a_{2} \frac{d \lambda}{d a_{1}}\right)\left(\square^{\prime}-y^{\prime} \partial_{x^{\prime}}\right) \phi
$$

et puisque $n a \frac{d \lambda}{d a_{0}}+(n-1) a_{2} \frac{d \lambda}{d a_{1}}$ se réduit à $n \frac{a_{1}^{2}}{a_{0}^{2}}-(n-1) \frac{a_{0} a_{2}}{a_{0}^{2}}$, ou enfin à $\lambda^{2}-\frac{(n-1) a_{2}^{\prime}}{a_{0}}$, ce terme devient

$$
\left(\lambda^{2}-\frac{(n-1) a_{2}^{\prime}}{a_{0}}\right)\left(\square^{\prime}-y^{\prime} \partial_{x^{\prime}}\right)
$$

Le terme $-x^{\prime} \partial_{y} \cdot \phi$ se réduit à $-\left(x^{\prime}+\lambda y^{\prime}\right)\left(-\lambda \partial_{x^{\prime}}+\partial_{y^{\prime}}\right) \phi$, savoir à

$$
\left(-x^{\prime} \partial_{y^{\prime}}+\lambda^{2} y^{\prime} \partial_{x^{\prime}}+\lambda x^{\prime} \partial_{x^{\prime}}-\lambda y^{\prime} \partial_{y^{\prime}}\right) \phi
$$

et en mettant pour un moment

nous obtenons

$$
\begin{array}{cc}
M= & n a_{1}\left(\partial_{a_{0}^{\prime}}+\lambda \partial_{a_{1}^{\prime}} \ldots \ldots+\lambda^{n} \partial_{a_{n}{ }^{\prime}}\right) \\
+(n-1) a_{2}( & \left.\partial_{a_{1}} \ldots \ldots+n \lambda^{n-1} \partial_{a_{n}{ }^{\prime}}\right) \\
\vdots & \left.\partial_{a_{n-1}}+n \lambda \partial_{a_{n^{\prime}}}{ }^{\prime}\right)
\end{array}
$$

$$
\left(\dot{\square}-x \partial_{y}\right) \phi=M \phi+\left(\lambda^{2}-\frac{(n-1) a_{2}^{\prime}}{a_{0}}\right)\left(\square^{\prime}-y^{\prime} \partial_{x^{\prime}}\right) \phi+\left(-x^{\prime} \partial_{y^{\prime}}+\lambda^{2} y^{\prime} \partial_{x^{\prime}}+\lambda x^{\prime} \partial_{x^{\prime}}-\lambda y^{\prime} \partial_{y^{\prime}}\right) \phi,
$$

c'est-à-dire

$$
\left(\dot{\square}-x \partial_{y}\right) \phi=\left(M-x^{\prime} \partial_{y^{\prime}}\right) \phi+\lambda^{2} \square^{\prime} \phi-\frac{(n-1) a_{2}^{\prime}}{a_{0}^{\prime}}\left(\square^{\prime}-y^{\prime} \partial_{x^{\prime}}\right) \phi+\lambda\left(x^{\prime} \partial_{x^{\prime}}-y^{\prime} \partial_{y^{\prime}}\right) \phi .
$$

Or en supposant que $\dot{\square}^{\prime}$ est ce que devient $\dot{\square}$ en y écrivant $a_{0}^{\prime}, a_{1}^{\prime}, \ldots a_{n}^{\prime}$ au lieu de $a_{0}, a_{1}, \ldots a_{n}$, et en posant

$$
\Theta^{\prime}=\left(0-\frac{1}{2} n\right) a_{0} \partial_{a_{0}^{\prime}}+\left(1-\frac{1}{2} n\right) a_{1}{ }^{\prime} \partial_{a_{1}^{\prime}}+\ldots\left(n-\frac{1}{2} n\right) a_{n}{ }^{\prime} \partial_{a_{n}}{ }^{\prime}
$$

on obtient, après avoir fait une réduction un peu pénible:

$$
M \phi+\lambda^{2} \square^{\prime} \phi=\dot{\square}^{\prime} \phi+2 \lambda \Theta^{\prime} \phi,
$$

(en effet les coefficients de $\partial_{a_{0}^{\prime}} \phi, \partial_{a_{1}} \phi \&$ c. aux deux côtés de sette équation deviennent les mêmes après des réductions convenables.) Done enfin on a

$$
\left(\dot{\square}-x \partial_{y}\right) \dot{\phi}=\left(\dot{\square}^{\prime}-x^{\prime} \partial_{y^{\prime}}\right) \phi-\frac{(n-1) a_{2}^{\prime}}{a_{0}^{\prime}}\left(\square^{\prime}-y^{\prime} \partial_{x^{\prime}}\right) \phi+2 \lambda\left(\Theta^{\prime}+\frac{1}{2} x^{\prime} \partial_{x^{\prime}}-\frac{1}{2} y^{\prime} \partial_{y^{\prime}}\right) \phi=0,
$$

ou bien, puisque cette équation doit être satisfaite indépendamment de la quantité $\lambda$ (qui seule contient $a_{1}$ ), elle se décompose dans les deux équations

$$
\begin{array}{r}
\left\{a_{0}^{\prime}\left(\dot{\square}^{\prime}-x^{\prime} \partial_{y^{\prime}}\right)-(n-1) a_{2}^{\prime}\left(\square^{\prime}-y^{\prime} \partial_{x^{\prime}}\right)\right\} \phi=0, \\
\left\{\Theta^{\prime}+\frac{1}{2} x^{\prime} \partial_{x^{\prime}}-\frac{1}{2} y^{\prime} \partial_{y^{\prime}}\right\} \phi=0,
\end{array}
$$


lesquelles, en y mettant d'abord $a_{1}^{\prime}=0$, puis en remettant $a_{0}, a_{2}, \ldots, a_{n}, x, y$ au lieu de $a_{0}^{\prime}, a_{2}^{\prime}, \ldots, a_{n}, x^{\prime}, y^{\prime}$, et en écrivant $\bar{\phi}, \bar{\Theta}, \cdot \square, \cdot \dot{\square}$ au lieu de $\phi, \Theta, \square, \dot{\square}$, donnent en effet les équations $(C, D)$ dont je me suis servi dans le texte.

\section{Note 2.}

Je vais résumer dans cette note quelques formules qui feront voir la liaison qui existe entre les invariants d'une fonction de $n$-ième ordre et de la fonction de $(n-1)$ ième ordre que l'on obtient en réduisant à zéro le coefficient de $y^{n}$, et en supprimant le facteur $x$.

Il convient pour cela de considérer une fonction telle que

$$
\left(a_{0}, a_{1}, \ldots a_{n}\right)(x, y)_{n}=a_{0} x^{n}+a_{1} x^{n-1} y \ldots+a_{n} y^{n},
$$

dans laquelle n'entrent plus les coefficients numériques du binôme $(1+x)^{n}$.

Écrivons

$$
\left(a_{0}, a_{1}, \ldots a_{n}\right)(x, y)_{n}=a_{0}\left(x-\alpha_{1} y\right)\left(x-\alpha_{2} y\right) \ldots\left(x-\alpha_{n} y\right)
$$

je tâche d'abord à représenter les invariants au moyen des racines $\alpha_{1}, \alpha_{2}, \ldots, \alpha_{n}$, et j'étends pour le moment le terme invariant à toute fonction, symétrique ou non, des racines qui ait la propriété caractéristique des invariants: fonctions qui jusqu'ici ont été considérées tacitement comme rationnelles par rapport aux coefficients.

Mettons d'abord

$$
\nabla=a_{0}^{2 n-2}\left(\alpha_{1}-\alpha_{2}\right)^{2}\left(\alpha_{1}-\alpha_{3}\right)^{2} \ldots\left(\alpha_{n-1}-\alpha_{n}\right)^{2} ;
$$

cette quantité $\nabla$ qui, égalée à zéro, exprime l'égalité de deux racines, et que je vais désormais nommer le Discriminant de la fonction, sera une fonction rationnelle des coefficients, et d'un invariant proprement dit. Mais de plus, toute fonction telle que $\left(\alpha_{1}-\alpha_{2}\right)^{n}\left(\alpha_{1}-\alpha_{3}\right)^{n}, \ldots$, dans laquelle la somme des indices des facteurs qui contiennent $\alpha_{1}$, celle des indices des facteurs qui contiennent $\alpha_{2}$, \&c. sont égales, sera un invariant; et en réunissant ces fonctions, pour trouver une somme en fonction symétrique des racines, on obtiendra des invariants proprement dits. Cela soit dit en passant. Pour le moment il suffit de prendre les invariants les plus simples, savoir ceux de la forme

$$
\frac{\left(\alpha_{1}-\alpha_{2}\right)\left(\alpha_{3}-\alpha_{4}\right)}{\left(\alpha_{1}-\alpha_{3}\right)\left(\alpha_{2}-\alpha_{4}\right)},
$$

lesquels en effet sont des rapports anharmoniques de quatre racines, prises à volonté. Soient $Q_{1}, Q_{2}, \ldots, Q_{n-3}$ la fonction qui vient d'être écrite et les fonctions que l'on en tire en mettant $\alpha_{5}, \alpha_{6}, \ldots, \alpha_{n}$ au lieu de $\alpha_{4}$. Les fonctions $\nabla, Q_{1}, Q_{2}, \ldots, Q_{n-3}$ seront des invariants indépendants, et le nombre de ces invariants est $n-2$. Donc, tout autre 
invariant sera une fonction des quantités $\nabla, Q_{1}, Q_{2}, \ldots, Q_{n-3}$. Soit maintenant $a_{n}=0$, et $\alpha_{n}$ la racine qui devient égale à zéro. Les quantités $Q_{1}, Q_{2}, \ldots, Q_{n-4}$ seront toujours des rapports anharmoniques de quatre racines de l'équation du $(n-1)$ ième ordre. Il n'y aura que la seule quantité $Q_{n-3}$ qui change de forme, et elle ne sera pas un invariant de la fonction du $(n-1)$ ième ordre. On voit aussi d'abord que le discriminant $\nabla$ se réduit à $a_{n-1}^{2} \nabla_{0}$, en exprimant par $\nabla_{0}$ le discriminant de la fonction du $(n-1)$ ième ordre. (C'est je crois M. Joachimsthal qui a le premier remarqué cette circonstance.) Donc, en supposant $a_{n}=0$, l'invariant de la fonction du $n$-ième ordre deviendra une fonction de $a_{n-1}^{2} \nabla_{0}, Q_{1}, Q_{3}, \ldots Q_{n-4}$ et d'une quantité $X$ qui n'est pas un invariant de la fonction du $(n-1)$ ième ordre, mais qui sera toujours la même quel que soit l'invariant dont il s'agit. En considérant les invariants proprement dits de la fonction du $(n-1)$ ième ordre, on peut former avec ces invariants des quotients $I_{1}, I_{2}, \ldots, I_{n-4}$ du degré zéro par rapport aux coefficients. Nous pouvons remplacer par ces quotients les quantités $Q_{1}, Q_{2}, \ldots, Q_{n-4}$, et dire que l'invariant de la fonction du $n$-ième ordre, en mettant $a_{n}=0$, deviendra une fonction des quantités $a^{2}{ }_{n-1} \nabla_{0}, I_{1}, I_{2}, \ldots, I_{n-4}$ et $X$.

Ces théorèmes auront, je crois, quelque utilité pour les recherches ultérieures: je les laisse à côté maintenant, et veux présenter une méthode assez simple pour calculer les discriminants.

Pour cela je remarque que les équations $(A)$, en changeant, comme nous venons de le faire, les valeurs des coefficients, donnent pour les invariants:

$$
\begin{array}{r}
\left(n a_{0} \partial_{a_{1}}+(n-1) a_{1} \partial_{a_{2}} \ldots+a_{n-1} \partial_{a_{n}}\right) \phi=0, \\
\left(a_{1} \partial_{a_{0}}+2 a_{2} \partial_{a_{1}} \ldots+n a_{n} \partial_{a_{n-1}}\right) \phi=0 ;
\end{array}
$$

et ces équations seront satisfaites en mettant pour $\phi$ le discriminant $\nabla$. Or, pour $a_{n}=0$, la fonction $\nabla$ devient $a_{n-1}^{2} \nabla_{0}$, ou, si l'on veut, $-a_{n-1}^{2} \nabla_{0}$; donc $\nabla$ sera généralement de la forme

$$
\nabla=-a_{n-1}^{2} \nabla_{0}+B a_{n}+C a_{n}^{2}+\ldots
$$

où $a_{n}{ }^{n-1}$ est la puissance la plus élevée de $a_{n}$. Donc, en supposant que $\nabla_{0}$ soit connu, et en mettant la première des équations écrites ci-dessus sous la forme $\left(F+a_{n-1} \partial_{a_{n}}\right) \nabla=0$, où $F=n a_{0} \partial_{a_{1}}+(n-1) a_{1} \partial_{a_{2}} \ldots+2 a_{n-2} \partial_{a_{n-1}}$ on obtiendra par la seule différentiation les coefficients $B, C$, \&c. En effet, cette équation donne

$$
a_{n-1} B=F\left(a_{n-1}^{2} \nabla_{0}\right), \quad 2 a_{n-1} C=-F(B), \quad 3 a_{n-1} D=-F(C) ;
$$

et ainsi de suite.

En supposant par exemple $n=3$, considérons la fonction du troisième ordre

$$
\alpha x^{3}+\beta x^{2} y+\gamma x y^{2}+\delta y^{3}:
$$

le discriminant de $\alpha x^{2}+\beta x y+\gamma y^{2}$ sera $4 \alpha \gamma-\beta^{2}$. Nous avons alors

$$
\nabla=-\gamma^{2}\left(4 \alpha \gamma-\beta^{2}\right)+B \delta+C \delta^{2}
$$

C. II. 
et en mettant $F=3 \alpha \partial_{\beta}+2 \beta \partial_{\gamma}, B, C$ seront donnés par

$$
\gamma B=F^{\prime}\left(4 \alpha \gamma^{3}-\beta^{2} \gamma^{2}\right), \quad 2 \gamma C=-F^{\prime}(B),
$$

c'est-à-dire $B=18 \alpha \beta \gamma-4 \beta^{3}, C=-27 \alpha^{2}$, et de là :

$$
\nabla=-27 \alpha^{2} \delta^{2}+18 \alpha \beta \gamma \delta-4 \alpha \gamma^{3}-4 \beta^{3} \delta+\beta^{2} \gamma^{2}:
$$

valeur qui correspond en effet à la forme ordinaire

$$
\nabla=-a^{2} d^{2}+6 a b c d-4 a c^{3}-4 b^{3} d+3 b^{2} c^{2},
$$

en changeant d'une manière convenable les coefficients.

Londres, Stone Buildings, 23 Févr. 1852. 